\title{
Discovering Drug Seeds by Practical NMR Strategies
}

\author{
Steven LaPlante \\ University of Quebec INRS-IAF, Canada
}

Small molecule drugs continue to be crucial for combating diseases. This presentation will convey the critical role NMR has been playing for discovering the seeds for new drugs starting from substrate peptides, high-throughput screens and fragment-based screens. Central to all the examples was the need to better understand the properties of small molecules when free in solution and to decipher the various types of binding to macromolecules. To do so, appropriate NMR methods/strategies were developed to prioritize quality ligands for downfield medicinal chemistry purposes. Examples will be shown where NMR strategies revealed compound solution behavior (solubility, aggregation, atropisomer chirality), exposed target protein features (folding and changes), and determined stoichiometric binding attributes (see Figures 1 and 2).

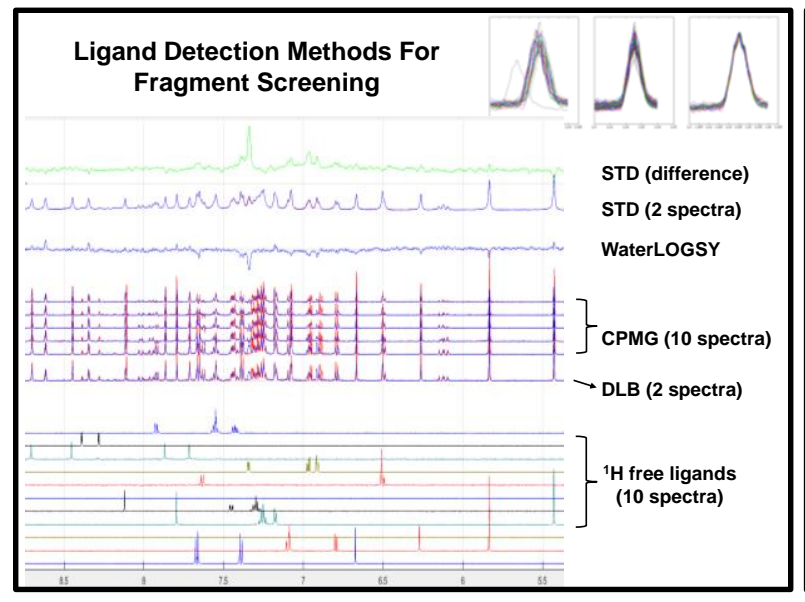

Figure 1. NMR ligand-detection methods.

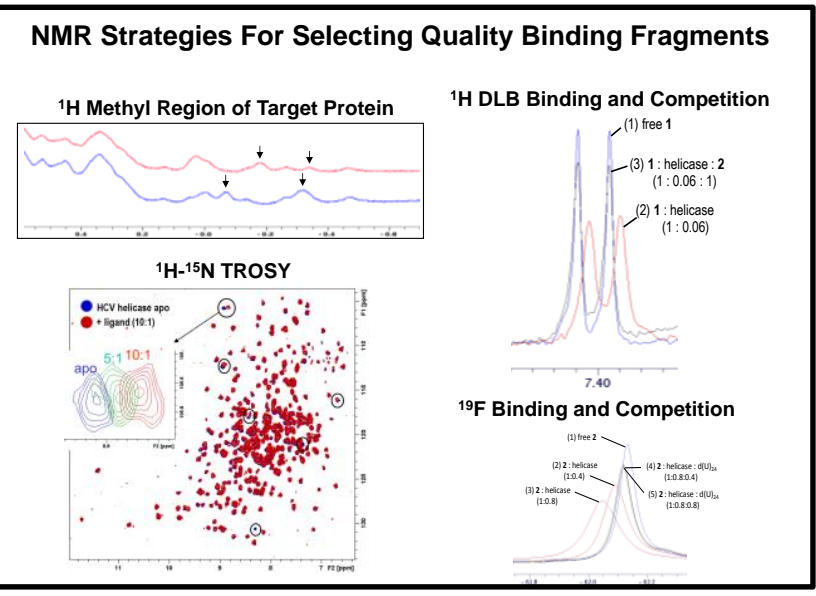

Figure 2. NMR methods for selecting stoichiometric ligand binding.

Acknowledgments: Harvard Medical School: G. Wagner, G. Heffron, P. Coote, H. Arthanari; NMX Research and Solutions Inc.: F. Bilodeau; Boehringer Ingelheim. 